\title{
Does age elevate blood pressure in obstructive sleep apnea?
}

\author{
Ma Wei $\cdot$ Chen Hui $\cdot$ Zhen-hua Gao
}

Received: 20 May 2012/ Accepted: 12 June 2012/Published online: 30 June 2012

(C) Springer-Verlag 2012

It was a great pleasure that we have read the article entitled "Combined effect of obstructive sleep apnea and age on daytime blood pressure" by Chao et al. [1]. After reading the article, we benefited a lot from it. We would like to share our opinions about the study. The following suggestions are necessary to be considered.

The article showed us there was a significant correlation between blood pressure and age in patients with mild-to-moderate obstructive sleep apnea (OSA). But the arterial hypertension and age were not correlated in the severe OSA subjects. This study presented the complicated relationship between OSA, age, obesity and arterial hypertension. It may be the reason that different key factors play the core part in different stages of development.

The prevalence of OSA has been shown to be around $50 \%$ in a general hypertension. Obstructive sleep apnea appeared to be the most common condition associated with resistant hypertension [2]. The strongest evidence supported an independent causal link between OSA and arterial hypertension.

The association between blood pressure variability and target organ damage, independently of absolute blood pressure values has been demonstrated. The prognostic

M. Wei · C. Hui · Z. Gao $(\bowtie)$

Graduate School of Tianjin Medical University,

Tianjin 300070, People's Republic of China

e-mail: tjgzh125@yahoo.cn

Z. Gao

Department of Cardiology, Tianjin Institute of Cardiology,

Second Hospital of Tianjin Medical University,

Tianjin 300211, People's Republic of China significance of blood pressure variability has lately enjoyed considerable attention [3]. In this study 1-day blood pressure had been recorded; why not analyze blood pressure variability?

In the study nocturnal polysomnography, questionnaire and physical examination were done. But the authors have not listed clear exclusion criteria. Other related disease and recent history of taking medicines should be considered, such as anti-hypertensive medications.

Body mass index has been as a marker of obesity, but the central adipose tissue may be more important in OSA. There was abundant data suggesting that measuring central obesity or total body fat content might be more appropriate than using the body mass index alone. If there is a chance, magnetic resonance imaging can be utilized to assess the extent of central obesity.

According to our experience, due to discomfort and fear, the subjects may have a bad sleep during nocturnal polysomnography, which could affect results.

Conflict of interest Nothing to declare.

\section{References}

1. Chao CC, Wu JL, Chang YT, Lin CY (2012) Combined effect of obstructive sleep apnea and age on daytime blood pressure. Eur Arch Otorhinolaryngol 269:1527-1532. doi:10.1007/s00405-011-1 $800-y$

2. Pedrosa RP, Drager LF, Gonzaga CC, Sousa MG, de Paula LK, Amaro AC et al (2011) Obstructive sleep apnea: the most common secondary cause of hypertension associated with resistant hypertension. Hypertension 58:811-817. doi:10.1161/HYPERTENSIONAHA.111.179788

3. Schutte AE, Schutte R, Huisman HW, van Rooyen JM, Fourie CM, Malan NT et al (2011) Blood pressure variability is significantly associated with ECG left ventricular mass in normotensive Africans: the SABPA Study. Hypertens Res 34:1127-1134. doi: 10.1038/hr.2011.104 